\title{
Channa striatus cream down-regulates tumour necrosis factor (TNF)-alpha gene expression and alleviates chronic-like dermatitis in mouse model
}

\begin{abstract}
Ethnopharmacological relevance:

Haruan, Channa striatus, is a freshwater fish which has been well-known locally to accelerate wound healing during post-operative and post-partum periods. The fish extract also has potent anti-inflammatory and analgesic properties.

Aim of the study:

To assess topical anti-inflammatory effect of Haruan cream on 12-0-tetradecanoylphorbol13-acetate (TPA)-induced chronic-like dermatitis in mice.

Materials and methods:

Male ICR mice were randomized into six groups of five mice each: acetone (vehicle), TPA alone (negative control), three Haruan treatment groups (Haruan 1\%, Haruan 5\% and Haruan $10 \%$ ) and hydrocortisone $1 \%$ (positive control). Briefly, both surfaces of mouse ears were applied with TPA $(2.5 \mu \mathrm{g} / 20 \mu \mathrm{l}$ acetone) for five times on alternate days and with Haruan or hydrocortisone $1 \%$ cream for the last three days. Mouse ear thickness was measured 24h after final treatment with the cream and the ears were harvested for further histological analysis and gene expression studies of TNF- $\alpha$ by real-time reverse transcriptase-polymerase chain reaction (RT-qPCR).

Results:

Topical application of Haruan cream had reduced the mouse ear thickness 18.1-28\%) with comparable effect to the positive control. In addition, histopathological comparison had shown evident reduction in various parameters of cutaneous inflammation including dermal oedema, inflammatory cells infiltration and proliferation of epidermal keratinocytes. Furthermore, TPA application had resulted in the up-regulation of TNF- $\alpha$ gene expression by 353 -fold, which was subsequently down-regulated by the Haruan cream (34- to 112-fold).
\end{abstract}

Conclusion:

Haruan is an effective topical anti-inflammatory agent in this mouse model of chronic-like dermatitis, thus suggesting its potential as a non-steroidal treatment option for chronic inflammatory dermatoses.

Keyword: Anti-inflammatory; Channa striatus; Chronic dermatitis; Gene expression; Haruan; TNF- $\alpha$ 